\title{
LAS TRANSFORMACIONES SOCIALES Y FÍSICAS POR SUBURBANIZACIÓN: EL CASO DE RINCÓN DE LA VICTORIA (MÁLAGA)*
}

\author{
Montosa Muñoz, J. y Corpas Alba, J. \\ Universidad de Málaga
}

\begin{abstract}
Resumen: El artículo que exponemos a continuación trata de describir los procesos de transformación físico y social que son inducidos desde un núcleo central, en este caso, el área objeto de estudio es la aglomeración urbana de Málaga, que acoge en su seno a una amalgama de municipios de diferentes ámbitos. En efecto, dentro de la aglomeración, podemos distinguir el entorno del litoral, con un mayor grado de integración en la dinámica urbana, en contraposición con el entorno del Valle y los Montes. En la costa oriental hemos seleccionado el municipio de Rincón de la Victoria, por ser un municipio que padece una intensa urbanización inducida desde la capital provincial y que se concreta con una serie de transformaciones físicas y sociales que trataremos de esbozar.
\end{abstract}

Palabras clave: Suburbanización, área metropolitana, neorrurales, transformaciones físicas por suburbanización, transformaciones sociales por suburbanización.

\begin{abstract}
The present report tries to explain the processes of change induced from a central city, in this case, the object of this study is Málaga Urban Agglomeration, that admits in its bossom an amalgam of municipalities from different fields. Inside the urban agglomeration, we really can distinguish the coast area, with a stage of integration in the urban dynamics, in contrast to the valley area and the Málaga mountains. On the Oriental Coast, we have selected the municipality of Rincón de la Victoria, because of the fact that it's a town that suffers from great changes due to a social group of urban procedence, eminently young and of middle qualification. These changes are what we ll try to explain.
\end{abstract}

Key words: Suburbanization, metropolitana area, newcomers, physical changes by suburbanization, social changes by suburbanization.

* Recibido: 10-11-03. Aceptado: 15-6-04. 


\section{Introducción}

A lo largo de la historia ha existido siempre una relación estrecha entre el campo y la ciudad. Tradicionalmente se ha visto que el origen de las transformaciones urbanas se ha producido en el campo. Gracias al excedente agrario, la población se pudo dedicar a tareas no exclusivamente agrarias, surgiendo actividades específicamente urbanas o que denominamos urbanas, como es el caso de la artesanía, o el comercio. Sin embargo, autores como Jane Jacobs defienden la tesis de que "las ciudades surgieron primero, y el desarrollo rural después" (Jacobs, 1970, p. 3-48). Esta idea aunque atrevida, permite constatar un hecho: que el papel difusor de la ciudad no nace en la revolución industrial, sino que se remonta a la misma génesis del hecho urbano. No obstante, y aquí trasluce la novedad, lo que es reciente es el cambio de un modelo de crecimiento urbano: de un crecimiento basado en un modelo concentrado de personas y recursos en espacios reducidos, a un modelo de crecimiento más disperso, hecho que ha caracterizado fundamentalmente a la sociedad postindustrial. Se forman así áreas urbanas en las que sobresale un núcleo central, que tiene el papel de difundir habitantes primero y más tarde actividades a su hinterland más próximo. $\mathrm{Al}$ conjunto de esta área se le ha venido en llamar área metropolitana. Es esta área metropolitana la que experimenta, en su fase inicial, un fenómeno de suburbanización que puede definirse como un conjunto de transformaciones inducidas desde la ciudad madre hacia las ciudades tributarias de su sistema urbano.

Las transformaciones comienzan con un tipo de residencia que es descrito por $\mathrm{K}$. Jackson ${ }^{1}$. Este mismo autor, a la hora de explicar el fenómeno histórico de la suburbanización de los Estados Unidos lo identifica con una clase media impulsada por una serie de factores, en primer lugar, la democratización de bienes que antes eran privativos de la clase social más alta como es el automóvil, que permite un hecho sin precedentes en la historia de la ciudad como es la disociación entre el lugar de trabajo y el de residencia. A este factor se unió la política de construir carreteras adecuadas que unieran los destinos y los centros emisores donde vivía la clase media norteamericana. Todos estos factores motivaron a que la población protagonizase un grado de transformación nunca conocido hasta entonces en el modelo de residencia: la democratización de la vivienda unifamiliar a la etnia blanca, de espacios privados que devoraban un enorme espacio antes rural y público. Así, las ciudades centrales fueron abandonadas por las clases medias, cuyo espacio fue ocupado por minorías étnicas que fomentaron la aparición de "slums" urbanos. La dimensión de este fenómeno se manifiesta elocuentemente a través de las cifras: si en 1950 el $67 \%$ de la población urbana norteamericana vivía en las ciudades centrales, en 1980 sólo representaban el $25 \%$ de la población.

También en Europa se produjo esta caída en el crecimiento de las ciudades centrales, aunque fue más tardía y de menor alcance que en Norteamérica. No obstante, 
y pese a la diferente morfología entre la ciudad norteamericana y la europea; desde la década de los años sesenta aparece una clara tendencia a la importación del modelo americano: la suburbanización en Europa, con unas características semejantes a las producidas en Norteamérica.

En definitiva, el suburbio de clase media constituye un espacio de gran importancia en el proceso reciente de urbanización y ha transformado el paisaje y el espacio social en las ciudades medias y grandes de los países desarrollados.

\section{La aglomeración urbana de Málaga²}

El documento que hemos empleado para abordar la idea de la aglomeración urbana de Málaga como un entorno que desborda los límites municipales de la ciudad central data de 1991, y es previo a la elaboración, por parte de la Junta de Andalucía, del primer documento que contempla la configuración oficial de una serie de aglomeraciones urbanas en Andalucía. Este documento distingue una primera corona de influencia directa, donde se incluían la ciudad central, Málaga; junto a los municipios de Benalmádena, Torremolinos, Alhaurín de la Torre, Alhaurín el Grande, Cártama, Almogía, Casabermeja, Totalán y Rincón de la Victoria. Los municipios anteriores constituyen el espacio donde se desarrolla, en términos oficiales, el fenómeno de área metropolitana.

La existencia de una entidad geográfica singular en las aglomeraciones urbanas de Andalucía resulta ser una característica general de las grandes aglomeraciones urbanas: la aglomeración urbana sevillana, en el Aljarafe o la aglomeración urbana granadina, en la Vega. No obstante, la aglomeración urbana malagueña no forma una entidad geográfica singular, ya que abarca parte del valle del Guadalhorce, parte del espacio litoral de la Costa del Sol, y, por último, los Montes de Málaga. Este heterogéneo territorio ha sido, de modo desigual, el soporte de la población y de las actividades económicas de un sistema de asentamientos con un débil grado de integración respecto a la ciudad madre. De este modo, como cita Feria Toribio, "únicamente Rincón de la Victoria y Alhaurín de la Torre, además de los núcleos de Churriana y Campanillas pertenecientes al mismo municipio de Málaga, constituyen ejemplos claros de la expansión metropolitana de esta ciudad".

La aglomeración urbana de Málaga presenta además algunas características que la diferencian de otras aglomeraciones: en Málaga no existe un sistema urbano dendrítico, como es puesto de manifiesto por Feria Toribio (Feria Toribio, 1992, p. 228), sino que la propia configuración física de su entorno, la dualidad espacial entre valle y litoral, y las dinámicas inducidas por la actividad turística en la Costa del Sol Occidental, han supuesto una alteración del modelo territorial que tiende a ser lineal, lo que se 
refleja principalmente en las relaciones entre la ciudad de Málaga y el espacio turístico del litoral, en contraposición a las relaciones ciudad-Valle o ciudad-Montes.

Una segunda característica de la aglomeración urbana de Málaga es el tamaño demográfico, por el que se sitúa, en orden de importancia, en el segundo lugar de Andalucía, tras el área metropolitana de Sevilla. El municipio de Málaga concentra la mayor parte de la población de la aglomeración, concretamente el 80\% de la población en 1992 (Feria Toribio, 1992, p. 220). Frente al extraordinario peso demográfico de la capital se opone la heterogeneidad poblacional de los municipios que integran la aglomeración, ya que mientras Torremolinos, Benalmádena, Alhaurín de la Torre y Rincón de la Victoria superan, algunos de ellos, ampliamente los 25.000 habitantes en el 2001, los municipios de los Montes y del Valle se caracterizan por su menor población, no superior en el caso de los Montes, a los 5.000 habitantes, indicador elocuente del distinto grado de urbanización del territorio y del mayor componente rural existente en dichos municipios.

De entre los núcleos del área, sobresalen dos: Alhaurín de la Torre, en el espacio próximo a la Vega del Guadalhorce y Rincón de la Victoria, en la costa, que aparecen en el contexto de la periferia como núcleos con un acelerado proceso de urbanización, fuertemente afectado por la impronta de población proveniente de la capital. Es en ellos donde con mayor intensidad se han producido transformaciones en las estructuras sociodemográficas y físicas; razones que nos han impulsado a estudiar la intensidad de estas transformaciones, seleccionando Rincón de la Victoria por su doble naturaleza de municipio turístico para la clase media malagueña y por su carácter de ciudad dormitorio de la capital.

\section{Las transformaciones sociales en Rincón de la Victoria}

Refiriéndonos a las transformaciones sociales vamos a citar dos hechos: el rejuvenecimiento de la población de Rincón de la Victoria y, en segundo término, los cambios en la estructura socioeconómica en relación, concretamente, al nivel de instrucción y a la cualificación profesional.

Para conocer la estructura por edad de la población inmigrante, hemos empleado el método de los supervivientes extraído de Courgeau ${ }^{4}$. Entendemos con ello que es toda la población inmigrante, no sólo la que procede de la capital. Sin embargo, conviene aclarar que la mayoría de la población inmigrante de Rincón de la Victoria proviene de la capital tal como se constata en trabajos anteriores. Los últimos datos disponibles al respecto apuntan que de los 14.371 inmigrantes registrados en el municipio entre 1991 y 2000 , en torno a 9.188 provenían de la capital, lo que representa un $63,93 \%$ del total de inmigrantes ${ }^{5}$, cifra que concuerda con otras fuentes ${ }^{6}$. 
Cuadro 1. Cálculo de la migración neta en Rincón de la Victoria (Método de los supervivientes).

\begin{tabular}{|c|c|c|c|c|c|c|}
\hline Grupos de edad & $\begin{array}{c}2001 \\
\text { Rincón }\end{array}$ & $\begin{array}{l}\text { Coeficiente de } \\
\text { supervivencia }\end{array}$ & Supervivientes & $\begin{array}{l}\text { Grupos } \\
\text { de edad }\end{array}$ & $\begin{array}{l}\text { Migración neta } \\
\text { de } 1991 \text { a } 2001\end{array}$ & $\begin{array}{l}\text { Resto de } \\
\text { la población }\end{array}$ \\
\hline De 0 a 4 & 1757 & 0,99814 & 1524 & De 0 a 4 & 233 & 1297 \\
\hline De 5 a 9 & 1530 & 0,99953 & 1165 & De 5 a 9 & 365 & 1165 \\
\hline De 10 a 14 & 1508 & 0,99786 & 1164 & De 10 a 14 & 344 & 1164 \\
\hline De 15 a 19 & 1667 & 0,99653 & 1130 & De 15 a 19 & 537 & 1130 \\
\hline De 20 a 24 & 1762 & 0,99657 & 1072 & De 20 a 24 & 690 & 1072 \\
\hline De 25 a 29 & 2202 & 0,99557 & 1074 & De 25 a 29 & 1.128 & 1074 \\
\hline De 30 a 34 & 2681 & 0,99516 & 1075 & De 30 a 34 & 1.606 & 1075 \\
\hline De 35 a 39 & 2553 & 0,99535 & 846 & De 35 a 39 & 1.707 & 846 \\
\hline De 40 a 44 & 2054 & 0,99248 & 739 & De 40 a 44 & 1.315 & 739 \\
\hline De 45 a 49 & 1520 & 0,98624 & 709 & De 45 a 49 & 811 & 709 \\
\hline De 50 a 54 & 1287 & 0,97585 & 599 & De 50 a 54 & 688 & 599 \\
\hline De 55 a 59 & 1254 & 0,96073 & 534 & De 55 a 59 & 720 & 534 \\
\hline De 60 a 64 & 1073 & 0,93472 & 508 & De 60 a 64 & 565 & 508 \\
\hline De 65 a 69 & 864 & 0,89359 & 355 & De 65 a 69 & 509 & 355 \\
\hline De 70 a 74 & 699 & 0,82106 & 221 & De 70 a 74 & 478 & 221 \\
\hline De 75 a 79 & 465 & 0,67856 & 286 & De 75 a 79 & 179 & 286 \\
\hline De 80 a 84 & 215 & 0,06730 & 0 & De 80 a 84 & 215 & 0 \\
\hline De 85 y más & 211 & 0,00000 & 0 & De 85 y más & 211 & 0 \\
\hline
\end{tabular}

Fuente: Instituto de Estadística de Andalucía. Censos de 1991 a 2001

Una vez comentada como característica de la inmigración en el municipio la procedencia mayoritariamente proveniente de la capital, se deduce que el método de Courgeau permite extraer el perfil del inmigrante, con lo que por extrapolación obtenemos una aproximación de la característica por edad de los neorrurales: su juventud.

La estructura por edad nos revela que la población que se establece en el municipio de Rincón de la Victoria es de generaciones jóvenes, de 25 a 45 años, parejas jóvenes, que elegirían el municipio como lugar de residencia. De este modo, los inmigrantes de 15 a 39 años representarian el 46\% del total de los inmigrantes, familias jóvenes que escogerían el municipio como lugar para establecer su vivienda familiar.

Ahora bien, ¿̇hasta qué punto es una información precisa el afirmar que si la población inmigrante es joven, la población neorrural, la originaria de la capital, también es joven?

Para responder a esta cuestión acudiremos a una encuesta realizada a la población del municipio en el verano de 1997 y dirigida por mí. Dicha encuesta fue realizada por muestreo aleatorio simple con un nivel de confianza del $95 \%$ y un margen de error de $\pm 5 \%$ a un total de 99 personas. En dicha encuesta discriminé a la población según su procedencia: nativos, inmigrantes de la capital, y resto de inmigrantes. Entre otros resultados, podemos distinguir la composición por edad según procedencia (cuadro 2 y subsiguientes). 
Cuadro 2. Composición por edad según procedencia: cabezas de familia e hijos.

\begin{tabular}{|lcc|}
\hline \multicolumn{1}{|c}{ Edades } & Nativos & Neorrurales \\
\hline De 40 a 60 años & $41,67 \%$ & $46,43 \%$ \\
Más de 60 años & $16,67 \%$ & $21,43 \%$ \\
No consta & $0 \%$ & $3,57 \%$ \\
Edad media & 45,71 años & 56,70 años \\
Sin hijos & $29,17 \%$ & $21,43 \%$ \\
Con hijos de 20 a 30 años & $16,67 \%$ & $42,86 \%$ \\
\hline
\end{tabular}

Fuente: Elaboración propia.

Como podemos apreciar, no es exacta la afirmación de que los neorrurales rejuvenecen a la población debido a su juventud, de hecho, la edad media de los cabeza de familia nativos encuestados no llegaba a los 46 años frente a los 56,7 de los cabeza de familia neorrurales. Sin embargo, el 42,86\% de las familias de los neorrurales tenían hijos de 20 a 30 años, frente al porcentaje del 17\% de los nativos. Es así que son los hijos de estos neorrurales los que contribuyen al proceso de rejuvenecimiento. Así pues, el rejuvenecimiento de la población se realiza gracias a los hijos de los inmigrantes de la capital, no a sus progenitores, los cuales tenderán a dejar su vivienda a sus hijos más jóvenes cuando éstos decidan establecerse como nuevas familias en el municipio. En este sentido, la hipótesis más factible teniendo en cuenta la contención del volumen de residentes temporales o, vulgarmente denominados veraneantes, es que el estatus de residente permanente se adquiera posteriormente al estatus de residente temporal, esto es, que se produzca una conversión de segunda residencia en residencia permanente, sobre todo de las parejas jóvenes, hijos de las generaciones que colonizaron previamente el espacio como residentes temporales (cuadro 3).

Cuadro 3.

Cálculo de la carga turística global del municipio de Rincón de la Victoria (1994-2003).

Población de derecho a 1-1-20037: 28.007 habitantes.

Producción de residuos sólidos urbanos a enero de $2003: 843.980 \mathrm{~kg} / 27 \mathrm{Tn} /$ día.

Producción de residuos sólidos urbanos a agosto de 2003: $1.882 .460 \mathrm{~kg} / 60,72 \mathrm{Tn} / \mathrm{dí}$.

Producción media per cápita y día: $0,9640 \mathrm{~kg}$ en 2003

Población total en temporada turística: $60.720 / 0,9640=62.988$ habitantes.

Población total de residentes temporales estables: $62.988-28.007=34.980$ residentes temporales estables.

Población media de 1994: 16.650 habitantes.

Producción de residuos sólidos urbanos en temporada baja: $22 \mathrm{Tn} /$ día.

Producción de residuos sólidos urbanos en temporada alta: $65 \mathrm{Tn} /$ día.

Producción media per cápita y día: $1,3213 \mathrm{~kg}$.

Población total en temporada turistica: $65.000 / 1,3213=49.194$ habitantes.

Población total de residentes temporales estables: 49.194-16.650=32.544 residentes temporales estables.

Fuente: Excmo. Ayuntamiento de Rincón de la Victoria. R.S.U. ${ }^{8}$ durante varios años. Callizo Soneiro 9. 
Desde el punto de vista de la cualificación socioprofesional de la población neorrural que llega al municipio, resaltar la gran diferenciación existente entre la población nativa, mayormente sin estudios, y la población que se instala en el municipio proveniente de la capital, con mayoría de estudios medios. En cuanto a los grupos profesionales, hay una clara diferenciación ya que, como citábamos, la suburbanización tiene como principales protagonistas a una población de clase media, de cualificación media, y dedicados a actividades eminentemente de servicios cualificados: son los denominados empleos de cuello blanco, white-collar en la terminología anglosajona. En contraposición, los trabajadores de cuello azul, trabajadores manuales cualificados, tienen una representación nada desdeñable entre los inmigrantes, el $45 \%$, pero se trata de obreros cualificados, a diferencia de los no cualificados de los nativos que suponen el $17,65 \%$ del total. No obstante, como expresa la Ponencia al II Plan Estratégico de Málaga, en todo caso se trata de "emigrantes que orientan sus actividades hacia la ciudad central en la que antes residian" (Plan Estratégico de Málaga 2010).

Comparativa del nivel de instrucción de la población según su origen en Rincón de la Victoria

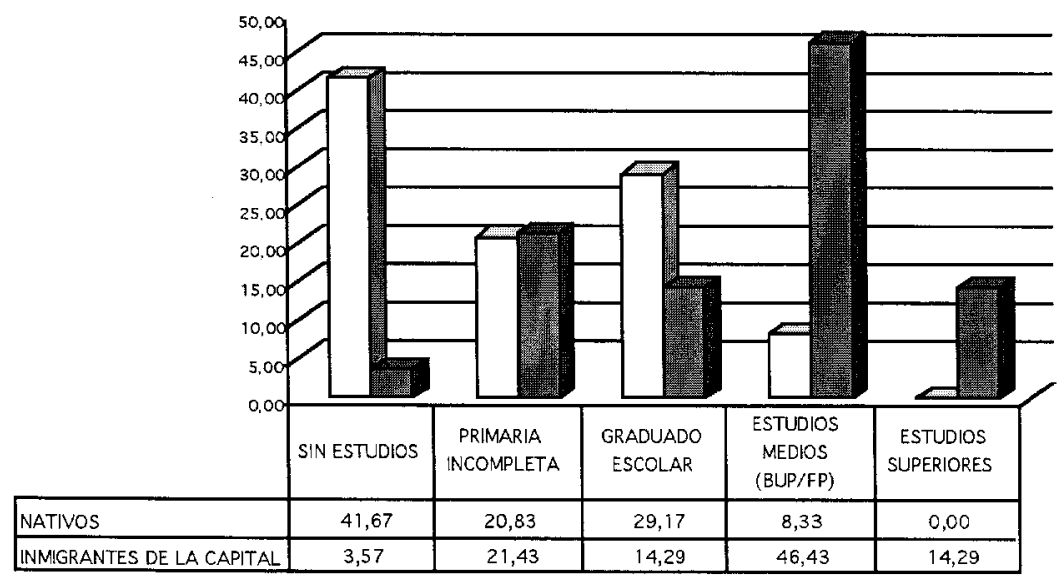

Gráfico 1.

Fuente: Elaboración propia a partir de datos de encuesta.

\section{Transformaciones físicas}

La repercusión que ha tenido la urbanización física en Rincón de la Victoria la hemos estudiado a partir de la superposición de la foto aérea de 1986 y la ortofoto de 2000, y la fuente para conocer los antiguos usos de los suelos que se han urbani- 
zado procede del Mapa de Cultivos y Aprovechamientos de la provincia de Málaga a escala 1:200.000 de 1986. De este modo hemos detectado zonas en el municipio que se han urbanizado más que otras. La pérdida de suelo rústico a suelo urbano ha supuesto un impacto visual y económico que se manifiesta en la reducción fundamentalmente de vegetación ligada a terrenos montañosos de pendientes significativas que estaban orientadas al monte leñoso que hemos identificado con el matorral y pastizal. Así, los suelos rústicos que suponen el paso de matorral-pastizal a tejido urbano, ha supuesto porcentajes significativos en Rincón de la Victoria, seguidos de La Cala del Moral, Norte de Rincón y Torre de Benagalbón. El suelo agrícola que se ha perdido ha tenido también una relevancia significativa, suponiendo valores superiores al 40 e incluso al 50\% en todas las entidades de población a excepción de Rincón de la Victoria (cuadro 3).

Cuadro 3. Relación de los porcentajes de pérdida de suelo rústico a urbano (estimación).

\begin{tabular}{|cccccc|}
\hline ZONAS & Improductivo & Matorral-Pastizal & Regadio & Secano & Ha \\
\hline CALA DEL MORAL & $0 \%$ & $53,6 \%$ & $6,5 \%$ & $39,9 \%$ & 1,45 \\
RINCON DE LA VICTORIA & $0 \%$ & $98,9 \%$ & $0 \%$ & $1,1 \%$ & 3,56 \\
TORRE DE BENAGALBÓN & $6,7 \%$ & $44,9 \%$ & $0 \%$ & $48,4 \%$ & 6,41 \\
AÑORETA & $20,20 \%$ & $7,10 \%$ & $0 \%$ & $72,70 \%$ & 2,69 \\
NORTE DEL RINCON & $0 \%$ & $50,3 \%$ & $0,4 \%$ & $49,3 \%$ & 6,25 \\
\hline
\end{tabular}

Fuente: Elaboración propia.

Cuantitativamente, el suelo que se ha urbanizado tiene un peso poco significativo si lo comparamos con la superficie total del municipio: se han urbanizado un total de 20,36 ha en 15 años, lo que representa el $0,74 \%$ de la extensión municipal, que es de 2.751 ha.

No obstante, si comparamos la superficie urbanizada del municipio entre 1986 y 2000, se evidencia un fuerte aumento de la superficie urbanizada: de 45,25 ha en 1986 se ha pasado a una zona urbanizada de $65,62 \mathrm{ha}$, lo que supone un crecimiento de la superficie urbanizada del municipio del $45,01 \%$, un porcentaje muy elevado, pero inferior al crecimiento demográfico que fue del $176,22 \%{ }^{10}$ en el intervalo de dichos años, lo que pone de manifiesto el papel destacado de la conversión de la segunda residencia en residencia permanente.

En números absolutos las pérdidas más significativas de suelo rústico corresponden a Torre de Benagalbón y a Rincón Norte, seguidas de lejos por Rincón de la Victoria; una zona surgida ex novo como Añoreta que es una urbanización ligada a un campo de golf, y, finalmente, La Cala del Moral.

Por categorías de uso, subrayar las pérdidas de vegetación natural de monte leñoso mediterráneo que representaba, según el Instituto de Estadística de Andalucía, un 
Cuadro 4. Suelo urbanizado entre 1986 y 2000 según su uso anterior.

\begin{tabular}{|c|c|c|}
\hline \multicolumn{3}{|l|}{ LA CALA DEL MORAL } \\
\hline Suelo urbanizado según su antiguo uso & Superficie (ha) & Porcentajes \\
\hline Regadío & 0,09 & 6,5 \\
\hline Secano & 0,58 & 39,9 \\
\hline Monte leñoso-Pastizal & 0,78 & 53,6 \\
\hline Improductivo & 0,00 & 0 \\
\hline TOTAL & 1,45 & 100 \\
\hline \multicolumn{3}{|l|}{ RINCÓN DE LA VICTORIA } \\
\hline Suelo urbanizado según su antiguo uso & Superficie (ha) & Porcentajes \\
\hline Regadío & 0,00 & 0 \\
\hline Secano & 0,04 & 1,1 \\
\hline Monte leñoso-Pastizal & 3,52 & 98,9 \\
\hline Improductivo & 0,00 & 0 \\
\hline TOTAL & 3,56 & 100 \\
\hline \multicolumn{3}{|l|}{ TORRE DE BENAGALBÓN } \\
\hline Suelo urbanizado según su antiguo uso & Superficie (ha) & Porcentajes \\
\hline Regadio & 0,00 & 0 \\
\hline Secano & 3,10 & 48,4 \\
\hline Monte leñoso-Pastizal & 2,88 & 44,9 \\
\hline Improductivo & 0,43 & 6,7 \\
\hline TOTAL & 6,41 & 100 \\
\hline \multicolumn{3}{|l|}{ AÑORETA } \\
\hline Suelo urbanizado según su antiguo uso & Superficie (ha) & Porcentajes \\
\hline Regadío & 0,00 & 0 \\
\hline Secano & 1,96 & 72,7 \\
\hline Monte leñoso-Pastizal & 0,19 & 7,1 \\
\hline Improductivo & 0,54 & 20,2 \\
\hline TOTAL & 2,69 & 100 \\
\hline \multicolumn{3}{|l|}{ RINCÓN NORTE } \\
\hline Suelo urbanizado según su antiguo uso & Superficie (ha) & Porcentajes \\
\hline Regadío & 0,03 & 0,4 \\
\hline Secano & 3,08 & 49,3 \\
\hline Monte leñoso-Pastizal & 3,14 & 50,3 \\
\hline Improductivo & 0,00 & 0 \\
\hline TOTAL & 6,25 & 100 \\
\hline
\end{tabular}

Fuente: Elaboración propia y Mapa de Cultivos y Aprovechamientos de 1986.

total de 770 ha en el municipio en el año 2000, cuando ha perdido, en menos de 15 años, 10,51 ha, más de un 1,3\% de la superficie de monte leñoso del municipio. Le sigue en importancia el cultivo de secano, fundamentalmente leñoso, que engloba cultivos de baja rentabilidad económica como el olivar de aceituna de aceite y el almendro (245 ha en el 2000 según el Instituto de Estadística de Andalucía), que ha perdido un total de 8,76 ha en el mismo período. En contraposición a la situación de 
sangría que están padeciendo el monte mediterráneo y el olivar y el almendro de secano; el regadío no ha perdido apenas superficie, 0,12 ha en el mismo intervalo, siendo un regadío orientado tanto al herbáceo de regadío (55 ha en el 2000), como al leñoso de regadío ( $85 \mathrm{ha}$ ), destacando en esta última categoría cultivos como el aguacate, el chirimoyo y otros tropicales.
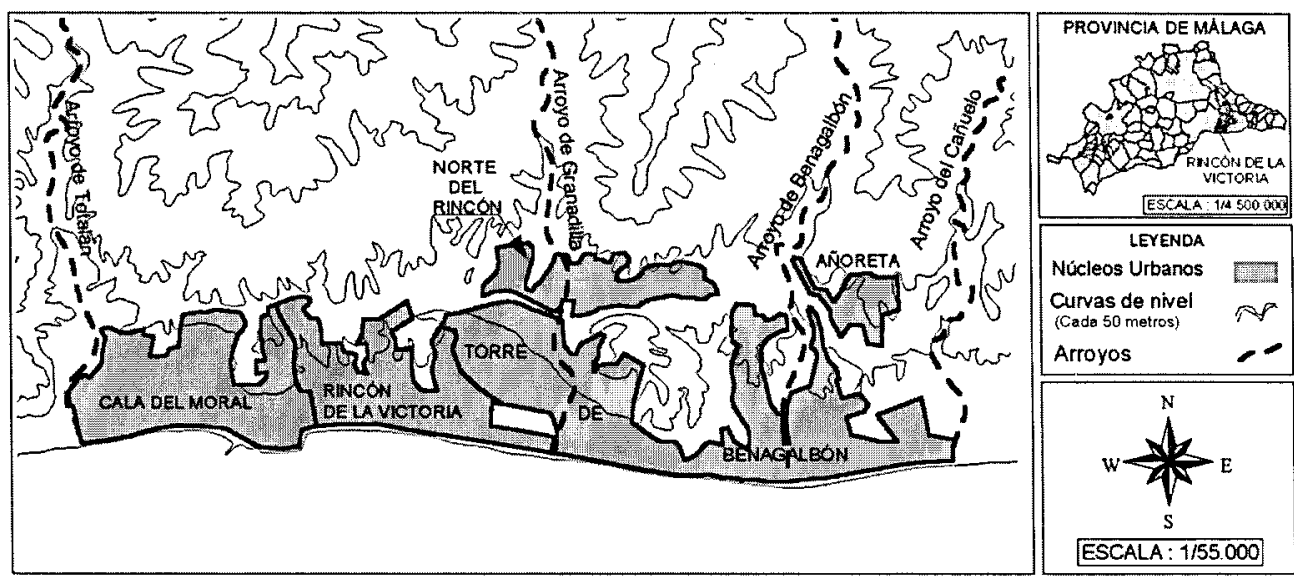

Mapa 1. Núcleos urbanos en Rincón de la Victoria en el año 2000. Fuente: Elaboración propia.
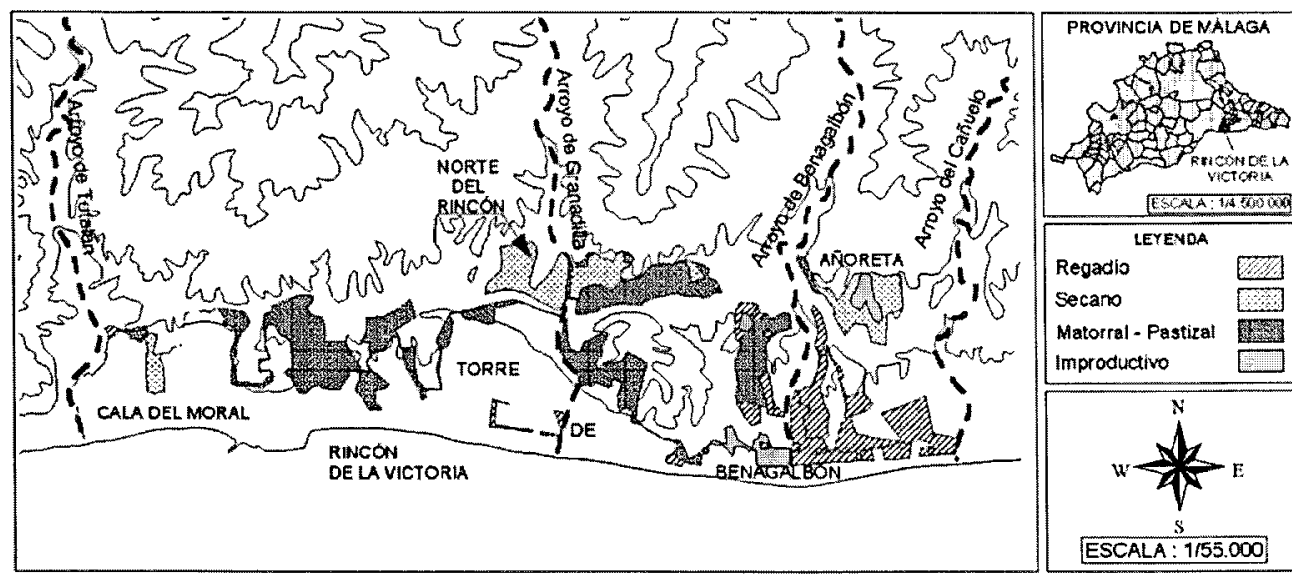

Mapa 2. Distribución por antiguos usos del suelo urbanizado en Rincón de la Victoria (1986-2000). 


\section{Conclusiones}

Una vez que hemos analizado la proliferación del espacio urbano en Rincón de la Victoria en el periodo 1986-2000 llegamos a las siguientes conclusiones:

1) La imagen geográfica transcurridos 15 años, denota una intensa transformación del espacio urbano: éste se ha incrementado considerablemente y se ha producido gracias a la reducción de espacios de monte leñoso y secano leñoso.

2) El incremento del espacio urbano durante el período de estudio es el resultado de un proceso de suburbanización, que ha importado población de extracción urbana de clase media, proveniente en su mayoría de la capital provincial.

3) Pese a todo, el crecimiento urbanizador no ha sido tan intenso como el crecimiento demográfico, gracias a la conversión de la segunda residencia en residencia permanente tal como se pone de manifiesto en el mantenimiento de las cifras de la población turística estable (34.980 en 2003 y 32.544 en 1994, crecimiento del $0,75 \%$ anual), frente a la población permanente de derecho (28.007 en 2003 y 16.650 en 1994 , crecimiento del 6,82\% anual); así como al desigual ritmo urbanizador existente entre el crecimiento urbanístico y el crecimiento demográfico.

4) La conversión de la segunda residencia en residencia permanente es el colofón a un proceso de colonización del territorio que se inició previamente con la ocupación temporal de las viviendas por parte de los progenitores y que culmina con las parejas jóvenes de los hijos que son los responsables, en última instancia, del rejuvenecimiento de la población del municipio.

5) Este proceso de suburbanización se produce sobre todo hacia el interior, ya agotado el suelo disponible en la franja costera, aunque en este sentido quisiéramos hacer mención a los intensos procesos de renovación urbana en detrimento de la vivienda local de los núcleos urbanos de Cala del Moral y Rincón de la Victoria, donde se ha producido (y nos consta por trabajo de campo), una renovación urbana intensa que ha transformado la morfología del centro del núcleo, con el paso de una vivienda autóctona en vivienda urbana.

Se corre el peligro de que las escasas zonas agrícolas o naturales del municipio sean pasto de la presión urbanística, una vez que las rentas agrícolas sean insufi- 
cientes o que el agricultor opte por vender a las constructoras. El resultado es un medio urbano con una fuerte presión antrópica, tanto en los frentes pioneros como en el centro urbano, lo que deja una herencia para el futuro de difícil solución, pues el crecimiento urbano adolece de falta de equipamientos, pese al volumen de viviendas, todo ello sin contar el valor de pérdida de paisaje y de deterioro visual de una urbanización exógena, ajena al municipio y parasitaria de él, un parasitismo que podría regenerarse si se llevara bajo el control de los poderes públicos en una simbiosis de relaciones entre la ciudad madre y la filial, entre el centro y la periferia urbana.

\section{Notas}

1 Jackson, K. T. (1985): Crabgrass Frontier. The suburbanization of the United States. Nueva York. Oxford University Press.

$2 \mathrm{El}$ documento base sobre el que nos fundamentamos se integra en el capítulo dedicado al análisis y diagnóstico del Plan de Ordenación Territorial de la Aglomeración urbana de Málaga. Consejería de Obras Públicas y Transportes. Junta de Andalucía, p. 10-26.

3 Feria Toribio, J. M. (1992): El sistema urbano andaluz. Instituto de Desarrollo Regional. Consejería de Obras Públicas y Transportes.

4 Courgeau, D. (1988). "Méthodes indirectes de mesure de la migration nette" en Méthodes de mesure de la mobilité spatiale. Migrations internes, mobilité temporaire, navettes. Paris. Éditions de L Institut National d'Etudes démographiques, p. 210-226.

5 Datos extraídos del Instituto de Estadística de Andalucía (Estadística de Variaciones Residenciales). Varios años.

6 Montosa Muñoz, J. (1997): Rincón de la Victoria. La población en un municipio metropolitano de Málaga. Universidad de Málaga.

7 No hemos podido obtener la cifra media de habitantes del 2003 al no estar disponible aún los datos. La población es a 1-1-2003, igual que la producción de residuos sólidos urbanos en temporada baja, que también corresponde al mes de enero.

8 Residuos Sólidos Urbanos.

9 Callizo Soneiro (1991): Aproximación a la Geografia del Turismo. Ed. Síntesis. Espacios y Sociedades. Madrid.

10 Instituto Nacional de Estadística: Cifras de población extraídas del Censo de 2001 y Padrón de 1986. 


\section{Bibliografía}

Callizo Soneiro (1991): Aproximación a la Geografia del Turismo. Ed. Síntesis. Espacios y Sociedades. Madrid.

COPT. JUNTA DE ANDALUCÍA. "Documento de análisis y diagnóstico de la Aglomeración urbana de Málaga". Plan de Ordenación Urbana de la Aglomeración urbana de Málaga.

COPT. INSTITUTO DE CARTOGRAFÍA DE ANDALUCÍA. Ortofoto de Rincón de la Victoria (2000) y foto aérea del municipio de Rincón de la Victoria (1985).

Courgeau, D. (1988): "Méthodes indirectes de mesure de la migration nette". Méthodes de mesure de la mobilité spatiale. Paris. Éditions de L'Institute National d'Etudes démographiques, p. 210-226.

Feria Toribio (1992). El sistema urbano andaluz. Instituto de Desarrollo Regional. Consejería de Obras Públicas y Transportes.
INSTITUTO DE ESTADÍSTICA DE ANDALUCÍA: Censos de 1991 y 2001. Mortalidad por edad de 1991 a 2001 (los datos de 1991 son extrapolados de los datos de mortalidad por edad provinciales).

INSTITUTO NACIONAL DE ESTADÍSTICA: Cifras globales de población del Padrón de 1986 y del Censo de 2001 (datos definitivos).

Jackson, K. Т. (1985): Crabgrass frontier. The suburbanization of United States. Oxford University Press. New York.

Jacobs (1970): The economy of cities. Vintage Books. A division of Random House. New York.

MAPYA (1986). Mapa de cultivos y aprovechamientos. Provincia de Málaga. Escala 1. 200.000 .

Montosa Muñoz, J. (1997): Rincón de la Victoria. La población en un municipio metropolitano de Málaga. Universidad de Mátaga. 